\title{
BabaoDan attenuates high-fat diet-induced non-alcoholic fatty liver disease via activation of AMPK signaling
}

\author{
Dandan Sheng ${ }^{1 \dagger}$, Shanmin Zhao ${ }^{1 \dagger}$, Lu Gao ${ }^{1 \dagger}$, Huifei Zheng ${ }^{1}$, Wenting Liu', Jing Hou', Yuxiang Jin ${ }^{1}$, Fei Ye', \\ Qiudong Zhao', Rong Li ${ }^{1}$, Naping Zhao ${ }^{2}$, Li Zhang ${ }^{2}$, Zhipeng Han ${ }^{1 *}$ and Lixin Wei ${ }^{{ }^{* *}}$ (D)
}

\begin{abstract}
Background: Babaodan (BBD), a traditional Chinese medicine, has been shown to have protective effects during liver injury and ameliorate liver disease progression, but little is known about its effect on non-alcoholic fatty liver disease (NAFLD). The aim of this study was to investigate the effects of BBD on obesity-induced NAFLD.

Methods: C57BL/6 J mice were fed with normal diet, high fat diet (HFD) or HFD + BBD for 8 weeks. Weights of all mice were recorded every 3 days. At the end of the experiments, the level of livers, kidneys and adipose tissues of each animal was weighed. Blood serum levels of alanine aminotransferase (ALT), aspartate aminotransferase (AST), total cholesterol (TC), triglyceride (TG), high density lipoprotein cholesterol (HDL-C) cholesterol, low density lipoprotein cholesterol (LDL-C), glucose and leptin were detected with appropriate test kits. Haematoxylin-eosin (HE), Masson trichrome and Oil Red $O$ staining of the liver were performed. We applied immunohistochemical analysis to investigate the expression of TNF- $a$, IL- 6 and leptin in liver tissue. The expression of genes related lipid anabolism (SREBP1-C, ACC, SCD-1, LXRa and CD36) and B-oxidation (CPT-1 and PPARa) in liver and adipose tissues was determined by RT-PCR. The expression of AMPK and p-AMPK was determined by western blot analysis.

Results: We found the weight of bodies and tissues (retroperitoneal fat pads, kidneys and livers) of mice fed with HFD + BBD were significantly lower than that of HFD-fed mice. And liver injury induced by HFD was relieved in mice treated with BBD, accompanied with significant reduction were observed in serum ALT/AST activities and alleviated pathological damage. The levels of glucose, TG, TC, HDL-C and LDL-C in the liver or serum were significantly decreased on HFD + BBD group compared with HFD group. Furthermore, BBD treatment reduced the level of TNF- $a$ and IL-6 induced by HFD. The level of leptin in the liver and serum were reduced in mice fed with HFD + BBD than that of HFD-fed mice. Several lipid synthesis genes (SREBP1-C, ACC, SCD-1, LXRa and CD36) were down-regulated and that of B-oxidation (CPT-1 and PPARa) up-regulated in HFD + BBD group compared with HFD group. In addition, BBD increased the expression of $\mathrm{p}-\mathrm{AMPK}$ compared with untreated HFD group, which suggested BBD improved the activation of AMPK pathway.
\end{abstract}

Conclusion: In summary, our results indicate that BBD has potential applications in the prevention and treatment of NAFLD, which may be closely related to its effect on lipid metabolism via activation of AMPK signaling.

Keywords: Babaodan, Non-alcoholic fatty liver disease, Lipid metabolism, AMPK pathway

\footnotetext{
*Correspondence: hanzhipeng0311@126.com; weilixin_smmu@163.com

${ }^{\dagger}$ Dandan Sheng, Shanmin Zhao and Lu Gao contributed equally to this

work

${ }^{1}$ Tumor Immunology and Gene Therapy Center, Third Affiliated

Hospital of Second Military Medical University, NO. 225 Changhai Road,

Shanghai 200438, China

Full list of author information is available at the end of the article
}

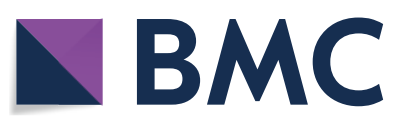

(c) The Author(s) 2019. This article is distributed under the terms of the Creative Commons Attribution 4.0 International License (http://creativecommons.org/licenses/by/4.0/), which permits unrestricted use, distribution, and reproduction in any medium, provided you give appropriate credit to the original author(s) and the source, provide a link to the Creative Commons license, and indicate if changes were made. The Creative Commons Public Domain Dedication waiver (http://creativecommons.org/ publicdomain/zero/1.0/) applies to the data made available in this article, unless otherwise stated. 


\section{Background}

Obesity is defined as an excessive body weight followed by accumulated adipose tissue [1]. A recent study showed that about 108 million children and 604 million adults are obese, and these numbers are on the rise [2]. Obesity is becoming one of the most serious public health problems worldwide. Moreover, obesity individuals have a high risk of numerous diseases, such as non-alcoholic fatty liver disease (NAFLD), a major chronic liver disease, which could lead to liver cirrhosis, liver cancer, and ultimately death [3]. NAFLD is frequently associated with increased visceral adiposity and metabolic abnormalities. A recent metaanalysis involving over 8.5 million individuals from 22 countries showed that more than $80 \%$ of NAFLD patients were overweight or obese, $72 \%$ had dyslipidemia $[1,4,5]$. The prevalence of NAFLD has increased in many countries. Approximately $25 \%$ of adults in the United States have fatty liver in the absence of excessive ethanol consumption [3]. In China, fatty liver disease is increasing at a rate of $0.594 \%$ per year and is expected to afflict $20 \%$ of Chinese by 2020 [6]. Therefore, NAFLD is becoming the most common liver disease worldwide. Therefore, great efforts have been continuously put into developing new therapeutic agents for obesity and NAFLD.

Traditional Chinese medicine (TCM) with a long history of clinical practices plays a crucial rule in disease treatment. For instance, Babaodan (BBD) as a classical TCM, has been showed to exert curative function in liver damage [7]. BBD has also been proved to be an adjuvant therapeutic strategy combining with chemotherapy in liver cancer, gastric cancer and lung cancer treatment [7]. Recently our laboratory has demonstrated that BBD ameliorated hepatic fibrosis through inhibiting activation of hepatic stellate cell and further protected liver from injury [8]. However, there is little evidence about BBD function exerted in obesity and associated diseases. In present study, we evaluated the effects of BBD on high fat diet-induced NAFLD.

\section{Methods \\ Animals}

Male C57BL/6 mice aged 6 weeks were purchased from the Shanghai Experimental Animal Center of the Chinese Academy of Sciences (Shanghai, China) and housed in maintained at the laboratory animal center of the Second Military Medical University. Experiments and procedures were approved by the Animal Ethics Committee of the Second Military Medical University. All animal handling and study procedures complied with the current Chinese regulation, GB14925-2010: Laboratory animal requirements of environment and housing facilities (Chinese version).

\section{Experimental design}

BBD was a gift from Shanghai Pharmaceutical Group Inc. All animals were acclimatized on a normal diet for 1 week and then divided into 3 study groups $(7$ mice/ group): (1) control group fed normal diet (ND, composed of $12 \%$-fat, $23 \%$-protein, $65 \%$-carbohydrates based on caloric content), (2) group fed high fat diet (HFD, composed of $40 \%$-fat, $20 \%$-protein, $40 \%$-carbohydrates based on caloric content), (3) group fed HFD + BBD ( $25 \mathrm{mg} / \mathrm{kg})$ and experimental period was 8 weeks. BBD was administered every 3 days by oral gavage for 8 weeks. At the end of feeding period, peripheral blood, liver, kidney and retroperitoneal fat pads were collected.

\section{Body and tissue weight}

Individual animals were weighed from the first BBD treatment every 3 days. The livers, kidneys and retroperitoneal fat pads of each animal were removed and weighed after the feeding experiment.

\section{Biochemical and ELISA analysis}

The concentrations of total cholesterol (TC), triglyceride (TG), high density lipoprotein cholesterol (HDL-C), cholesterol, low density lipoprotein cholesterol (LDL-C), alanine aminotransferase (ALT), aspartate aminotransferase (AST) and glucose in serum were tested with a biochemical auto-analyzer (Fuji Medical System, Tokyo, Japan) according to the manufacturer's instructions. Tumor necrosis factor $\alpha$ (TNF- $\alpha$ ), Interleukin 6 (IL-6) and leptin were measured with kits obtained from Abcam.

\section{Histopathological analysis}

The tissue specimens were embedded in paraffin wax, sliced to $4 \mu \mathrm{m}$ thickness, and placed on separate glass slides. After hematoxylin-eosin (HE) staining, Oil Red O staining and Masson trichrome staining, respectively, the sections were observed by light microscope.

\section{Immunohistochemical analysis}

For immunohistochemistry staining of TNF- $\alpha$, IL- 6 and leptin in liver tissue sections were deparaffinized in xylene and dehydrated with graded ethanol. After washing with $\mathrm{dH}_{2} \mathrm{O}$, tissue peroxidase was blocked with $3.0 \%$ hydrogen peroxide in methanol for $20 \mathrm{~min}$ at room temperature. For antigen retrieval using citric acid buffer, the slides were heated at $120{ }^{\circ} \mathrm{C}$ for $20 \mathrm{~min}$ and then cooled for $20 \mathrm{~min}$ at room temperature. After incubated overnight with the primary antibody IL-6 (1:800, Abcam, UK), TNF $\alpha$ (1:200, Abcam, UK) and leptin (1:500, Abcam, UK), and then with peroxidase-conjugated streptavidin for $15 \mathrm{~min}$. Diaminobenzidine-tetrahydrochloride 
(DAB) was used as the substrate to detect antigen-antibody binding. Sections were counterstained with hematoxylin and observed under the microscope.

\section{Real time-PCR}

To test mRNA expression, total RNA from every frozen mouse livers tissue was extracted by using TRIZOL reagent (Invitrogen, Carlsbad, CA, USA). Prime Script RT reagent Kit (Takara, Kyoto, Japan) was performed for cDNA synthesis. Relative quantitative PCR (RT-PCR) was performed using SYBR Green PCR Kit (Applied Biosystems, Foster City, CA, USA) according to the manufacturer instructions. The level of mRNA was expressed as the ratio of the signal intensity for each gene relative to that of GAPDH. Primer sequences are listed in Table 1.

Table 1 Primer sequence for real-time polymerase chain reaction

\begin{tabular}{ll}
\hline Gene & Primer sequence \\
\hline ACC & \\
F & CCGTTGGCCAAAACTCTGGAGCTAA \\
R & GAGCTGACGGAGGCTGGTGACA \\
LXR-a & \\
F & CTCAATGCCTGATGTTTCTCCT \\
R & TCCAACCCTATCCCTAAAGCAA \\
SCD-1 & \\
F & CGGTCATCCCATCGCCTGCTCT \\
R & GTAGGCGAGTGGCGGAACTGC \\
SREBP1-C & \\
F & GTGAGGCGGCTCTGGAACAGAC \\
R & ATAGGGGGCGTCAAACAGGCC \\
CD36 & \\
F & CACAGCTGCCTTCTGAAATGTGTGG \\
R & TTTCTACGTGGCCGGTTCTAATTC \\
PPARa & \\
F & ACTGGTAGTCTGCAAAACCAAA \\
R & AGAGCCCCATCTGTCCTCTC \\
CPT-1 & \\
F & TCTAGGCAATGCCGTTCAC \\
R & GAGCACATGGGCACCATAC \\
GAPDH & \\
F & GGCCCAGCAAGGACACTGAGTGTATTATTATGGGGGT \\
R &
\end{tabular}

\section{Western blot analysis}

The fresh liver tissue was washed with PBS and lysed by RIPA and PMSF at a ratio of 100:1. The insoluble material was removed by centrifugation $(12,000 \mathrm{~g})$ for $20 \mathrm{~min}$ at $4{ }^{\circ} \mathrm{C}$. The protein concentration in the supernatant was determined by BCA Protein Assay Kit (Beyotime, Jiangsu, China). Equal amount of proteins was separated by SDS-PAGE and western blot analysis. The glyceraldeyde-3-phosphate dehydrogenase (GAPDH) antibodies were obtained from Bioworld Technology (AP0063). The total AMP activated protein (AMPK) (5832 s), phosphoAMPK (p-AMPK) (2535S), antibodies were obtained from Cell Signaling Technology (CST, Boston, USA).

\section{Statistical analysis}

Results are presented as mean \pm SD. Statistical differences between groups were analyzed by the Student's $t$ test. Statistical tests of data were performed using the GraphPad Prism6 software (San Diego, CA, USA). $p<0.05$ was considered statistically significant.

\section{Result \\ BBD ameliorates lipid accumulation of HFD-induced obesity mice}

In order to investigate BBD effects on obesity, we conducted the model of obesity mice with HFD for 8 weeks. We found the weight of HFD mice was higher than that of normal diet group (Fig. 1a). Administration of BBD significantly decreased the weight gain compared to the high-fat group from the seventh week (Fig. 1a). The weight gained in the HFD + BBD group was significantly lower than that of HFD group at the end of feeding period (Fig. 1b). Meanwhile, retroperitoneal fat pads, kidneys and livers of mice fed with HFD + BBD were significantly lower than that of HFD-fed mice (Fig. 1c). We observed HFD-fed mice have more fat in retroperitoneal fat pads and perirenal fats compared with normal diet group and HFD + BBD group (Fig. 1d). The liver color of the high-fat control group appeared light yellow and that the normal group was dark red, while the liver color of HFD + BBD changed dark red (Fig. 1d). Furthermore, the epidydimal adipocyte size in HFD + BBD group was also lower compared with the HFD group (Fig. 1e, f). Taken together, these results demonstrate that BBD administration effectively prevents fat accumulation.

(See figure on next page.)

Fig. 1 BBD ameliorates lipid accumulation of HFD-induced obesity mice. a Changes in body weight in C57BL/6 mice fed normal diet ( $n=7)$, HFD $(n=7)$ or HFD + BBD $(n=7)\left(H F D+B B D-f e d\right.$ mice ${ }^{*} p<0.05$ vs. HFD-fed mice). b Body weight gain of different groups. $\mathbf{c}$ Weight of retroperitoneal fat pads, kidneys and livers in mice of different groups. $\mathbf{d}$ Representative pictures of retroperitoneal fat pads, kidneys and livers from different groups. $\mathbf{e}$ The cell sizes of the epididymal adipose tissues for HE-staining are shown. $\mathbf{f}$ Quantification of adipocyte size of different groups. Error bars reflect SD, ${ }^{*} p<0.05,{ }^{* *} p<0.01$ 

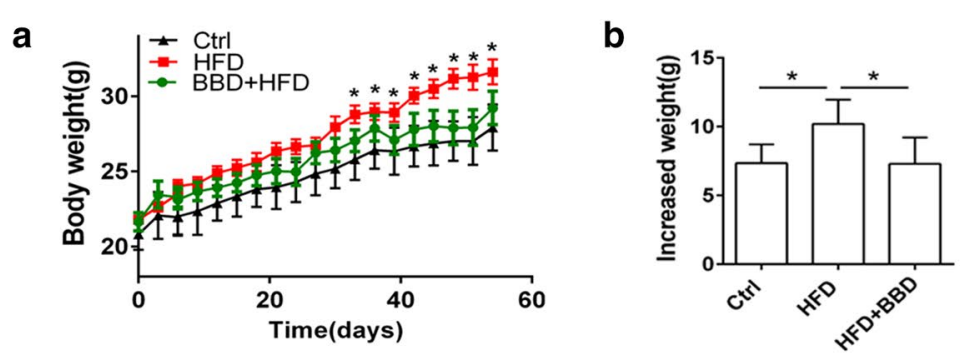

c
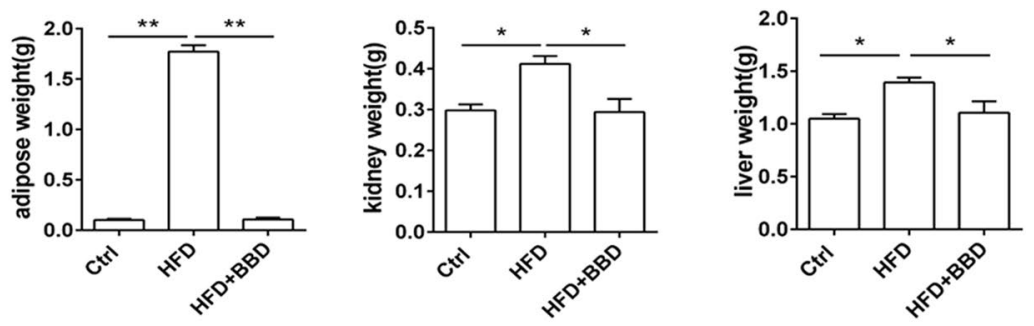

d
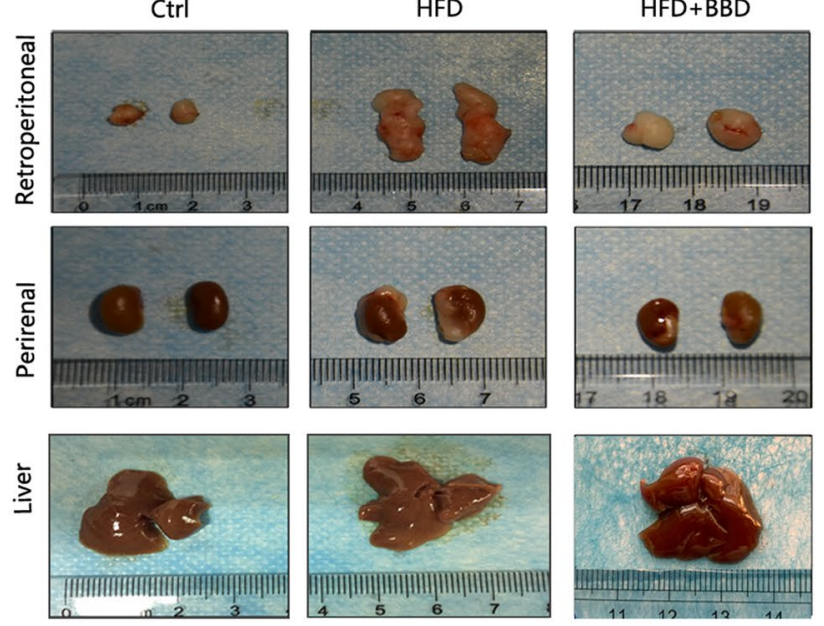

e
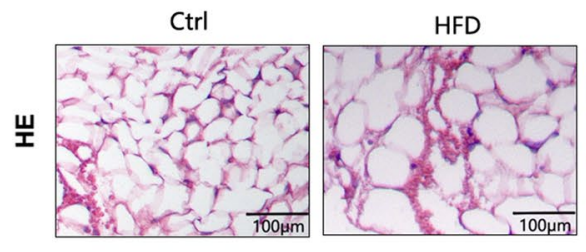

$H F D+B B D$

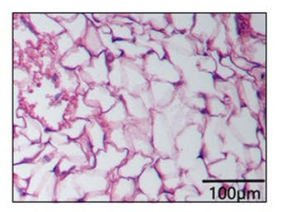

f

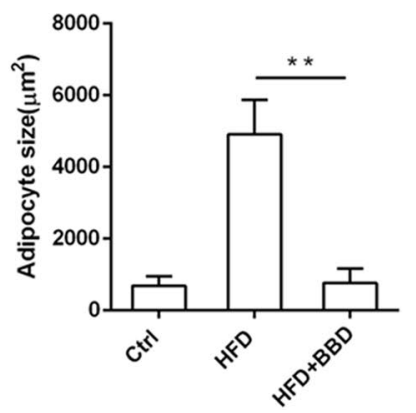




\section{$B B D$ relieves liver injury induced by high fat diet}

We detected HFD leaded to obvious liver injury in mice, as indicated by elevation of serum ALT and AST activities (Fig. 2a) and severe pathological damage (Fig. 2b, c). It was showed that hepatic fat accumulation in HFD group was obviously more than that in normal diet group. This suggested that long-term consumption of a high fat diet leads to the development of nonalcoholic fatty liver disease (NAFLD) in mice. To examine whether BBD affected liver injury induced by HFD, liver function indicators, morphological changes and the lipid contents in the liver of mice fed with HFD+BBD was detected. Histopathological analyses showed that BBD treatment relieved liver injury induced by HFD, accompanied with significant reduction in serum ALT/AST activities (Fig. 2a) and alleviated pathological damage (Fig. 2b). The HFD-group exhibited extensive liver bridging fibrosis and substantial collagen deposition (Fig. 2c). However, the HFD + BBD group had less bridging fibrosis and collagen (Fig. 2c). Meanwhile, BBD ameliorated the hepatic lipid droplets compared with no-treatment HFD mice (Fig. 2d). Additionally, BBD decreased the level of cholesterol and triglyceride in livers (Fig. 2e). As shown in Fig. 2f, the serum levels of glucose, TG, HDL-C and LDL-C were significantly decreased in the HFD + BBD group compared with the HFD group. And the serum TC of the HFD+BBD group was lower than that of the HFD group, although the difference was not statistically significant (Fig. 2e). Taken together, these results clearly demonstrate that BBD administration effectively relieve liver injury induced by high-fat diet through preventing hepatic fat accumulation.

\section{BBD relieves liver inflammation induced by high fat diet}

As obesity is a chronic inflammation that could cause damage to the liver, the liver inflammation was examined in each group. The expression of tumor necrosis factor $\alpha$ (TNF- $\alpha$ ) and Interleukin-6 (IL-6) were detected to access the role of BBD in inhibition of inflammation. As Fig. 3a showed, the serum levels of TNF- $\alpha$ and IL-6 were elevated in HFD group compared with normal diet group. BBD treatment reduced the serum levels of TNF- $\alpha$ and IL- 6 induced by HFD (Fig. 3a). Additionally, the expression of TNF- $\alpha$ and IL- 6 in liver analyzed by immunohistochemically staining also demonstrate that the inflammation induced by high fat diet were reduced by BBD (Fig. 3b). In conclusion, BBD ameliorated obesity-induced liver inflammation.

\section{BBD activates AMPK pathway to suppress expression of hepatic genes involved in lipogenesis}

With the accumulation of fat pad in the body, adipose tissue will release leptin to suppress the produce of fat [9-11]. The leptin level in serum and liver tissue of in HFD group mice was significantly elevated, while BBD treatment group could suppress leptin expression (Fig. 4a, b). To explore the molecular mechanisms of $\mathrm{BBD}$ regulates hepatic fat accumulation on high fat diet induced mice, we detected the expression of genes involved in lipogenesis (sterol regulatory element-binding protein 1c (SREBP1-c), acetyl coenzyme A-carboxylase (ACC), stearoyl-CoA desaturase-1 (SCD-1), liver $\mathrm{X}$ receptor alpha $(\mathrm{LXR} \alpha)$ and fatty acid translocase (FAT/CD36)) in the liver and adipose tissues by RTPCR. It was showed that the expression of SREBP1-C, ACC, SCD-1, LXR $\alpha$ and CD36 was significantly higher in HFD-fed mice than in the normal diet mice, but returned to basal levels in the BBD administrated mice (Fig. 4c). The gene expression related to $B$-oxidation (PPAR- $\alpha$, CPT-1) was down-regulated in HFD-fed mice and returned to basal levels in the $\mathrm{BBD}$ administrated mice (Fig. 4c). BBD moderately regulated the expression of these genes of BBD + HFD group compared with untreated HFD group, suggested that protective effects of BBD on HFD-induced obesity and NAFLD may be attributed to regulating the expression of these genes related with lipid metabolism.

Previous study had showed that the activation of AMPK signaling is involved in hepatic lipid metabolism by inhibiting the activity of SREBP-1c, ACC and others [12]. To further confirm whether AMPK is involved in the regulation effects of $\mathrm{BBD}$ against increased HFDinduced lipid synthesis, we detected the expression of AMPK and p-AMPK in livers. We found the expression of p-AMPK was decreased in HFD group (Fig. 4d), which indicated AMPK pathway were inactivation. Meanwhile, BBD increased the expression of p-AMPK compared with untreated HFD group (Fig. 4d). These data suggest that BBD regulated lipogenesis by activating AMPK pathway.

(See figure on next page.)

Fig. 2 BBD relieves liver injury induced by HFD. a Activity of ALT and AST in serum of mice fed normal diet, HFD or HFD + BBD; Representative images of $\mathbf{b} \mathrm{H} \& \mathrm{E}$, $\mathbf{c}$ Masson' trichrome and $\mathbf{d}$ oil-red $\mathrm{O}$ staining of the liver sections of different groups. e Relative cholesterol (TC) and triglyceride (TG) level in livers of different groups. $\mathbf{f}$ The level of glucose, total cholesterol (TC), low-density lipoprotein cholesterol (LDL-C), triglyceride (TG), high-density lipoprotein cholesterol $(\mathrm{HDL}-\mathrm{C})$ in serum of different groups. The data were shown as mean $\pm \mathrm{SD}$. $\mathrm{n}=7$ for all groups. ${ }^{*} p<0.05$ 
Sheng et al. Cell Biosci

(2019) 9:77

Page 6 of 10
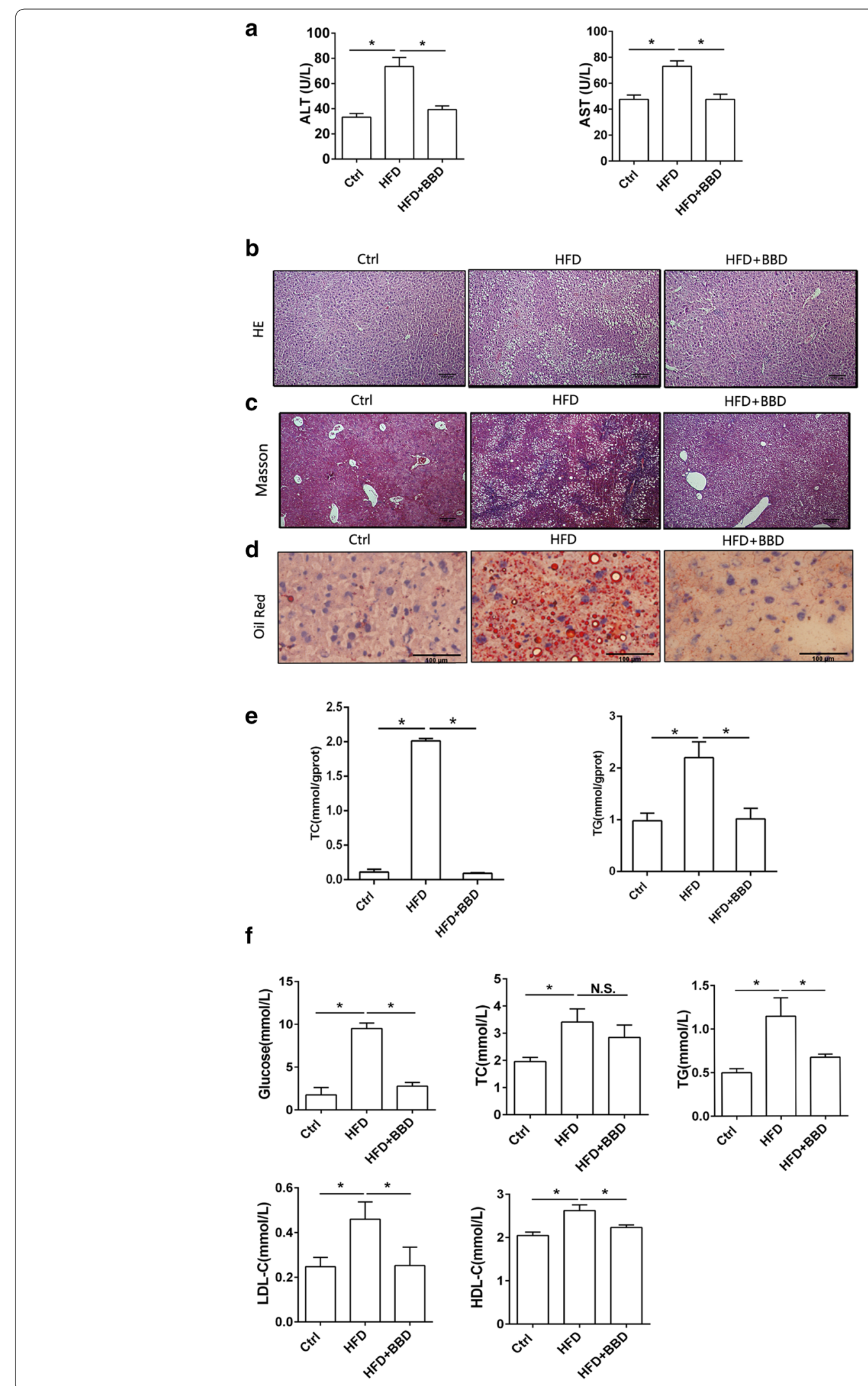
a
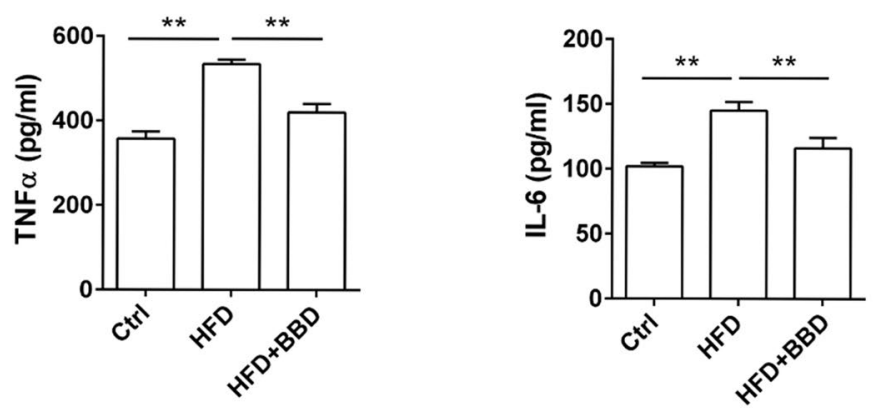

b

b

Ctrl

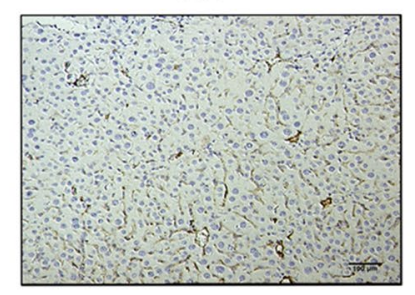

IL-6

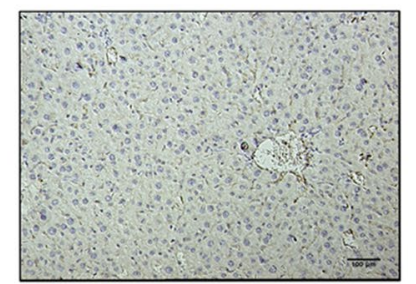

HFD
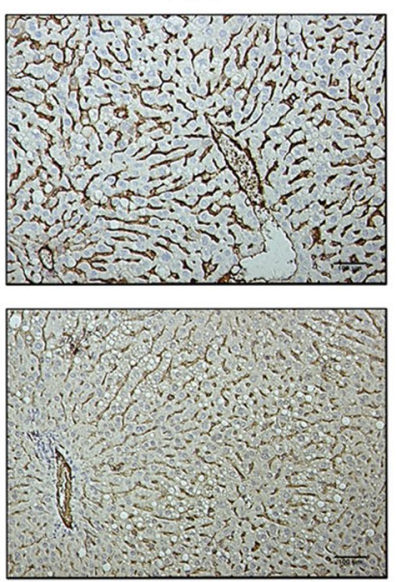

$H F D+B B D$
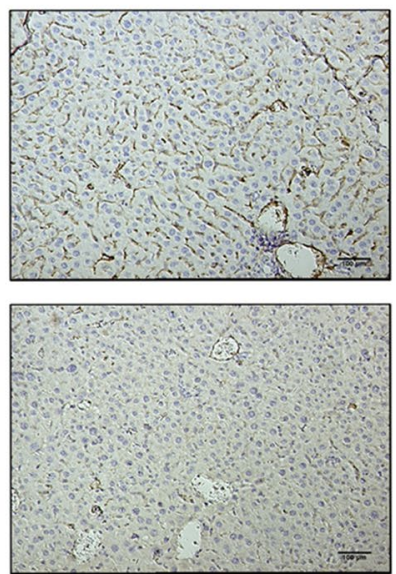

Fig. 3 BBD inhibits liver inflammation induced by HFD. a The concentration of TNF-a and IL-6 in serum of mice fed normal diet, HFD or HFD + BBD; b representative immunohistochemistry staining for TNF- $a$ and IL-6 in liver tissues. The data were shown as mean \pm SD. $n=7$ for all groups. ${ }^{* *} p<0.01$

\section{Discussion}

Babaodan (BBD) functions mainly by clearing heat and resolving dampness, removing blood stasis, dissipating mass and relieving pain [7]. Our former study indicated that BBD ameliorated hepatic fibrosis through inhibiting activation of hepatic stellate cell and further protected liver from injury [8]. In the present study, we observed that BBD attenuates HFD-induced obesity and NAFLD. It is showed that the circulating non-esterified fatty acids pool plays an important role in the pathogenesis of NAFLD and especially the overproduction of fatty acids in adipose tissues that flow to the liver [13]. We observed the BBD treatment ameliorated the body weights and weights of liver, kidney and inguinal subcutaneous fat in mice fed high fat diet. Meanwhile, BBD significantly decreased the fat weight and adipocyte size compared with HFD mice. In addition, BBD decreased both the serum levels of glucose, TG, HDL-C and LDL-C and hepatic TG, TC in BBD group. These results suggest that BBD alleviated the progression of obesity and NAFLD.

Adipose tissue is not only consisting of adipose cells, but also including macrophages closely related to inflammation. Previous studies had reported that obesity could increase local inflammation of fat and systemic inflammation, while weight loss reduced the systemic inflammation [14, 15]. Hotamisligil et al. [16] has pointed out that the nature of obesity is a systemic chronic low inflammation induced by various inflammatory factors. According to a study, adipose tissue macrophages increase from $\sim 10 \%$ of white adipose tissue cellularity in lean animals to up to $50-60 \%$ in the obese $[17,18]$. To investigate whether the relive effect of BBD could alleviate inflammation, we investigated the change of inflammation. Macrophages are major inflammatory and immune effector cells that play crucial roles in producing pro-inflammatory cytokines, such as tumor necrosis factor $\alpha$ (TNF- $\alpha)$ and interleukin 6 (IL-6) $[19,20]$. In this study, we found BBD administration could suppress the expression of TNF- $\alpha$ and IL- 6 . Thus, BBD ameliorated obesity-induced liver inflammation, which may be one of the reasons for alleviating liver injury in NAFLD.

Leptin is an adipocyte-derived cytokine that suppresses appetite and increases energy expenditure [21-23]. With fat accumulation in NAFLD patients, adipose tissue will 

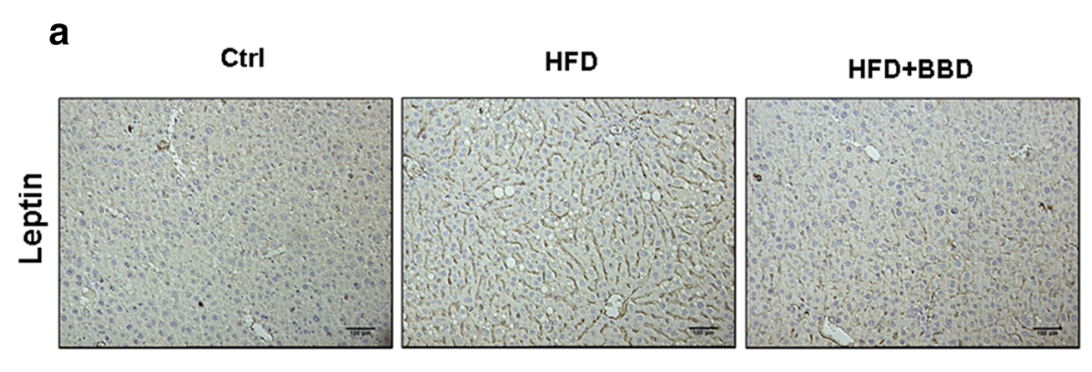

\section{b}
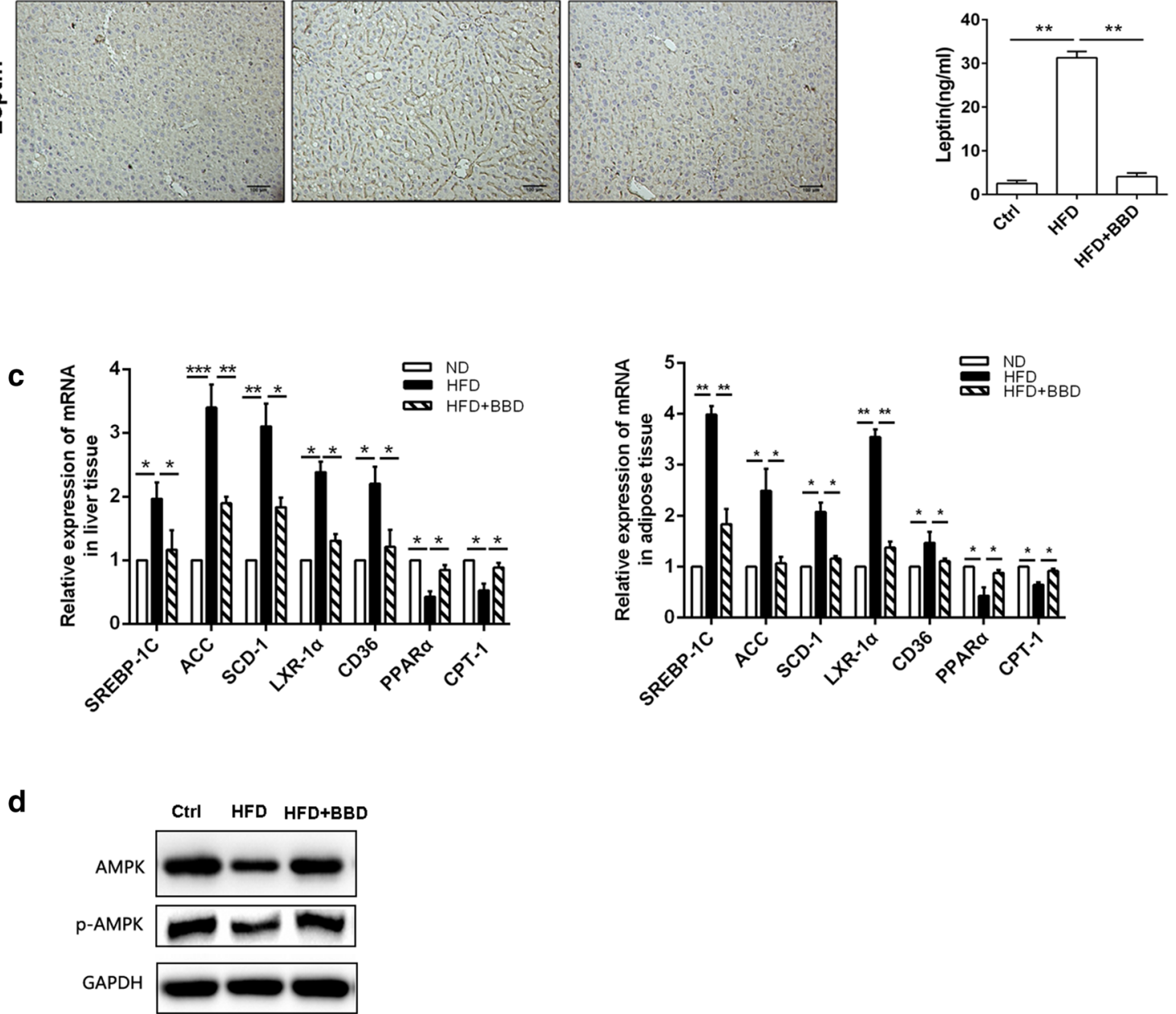

Fig. 4 BBD activates AMPK pathway to regulate hepatic gene expression involved in lipid metabolism. a Concentration of Leptin in portal vein serum was detected by Leptin Elisa test kit. b Representative immunohistochemistry staining for leptin in liver tissues of each group. c The expression of hepatic genes (SREBP1-C, ACC, SCD-1, LXRa, CD36, CPT-1 and PPARa) involved in lipogenesis or $\beta$-oxidation in liver and adipose tissues of different groups. $\mathbf{d}$ Western blot was used to detect the expression of AMPK and p-AMPK in livers of each group. The data were shown as mean \pm SD. $n=7$ for all grou ps. ${ }^{*} p<0.05,{ }^{* *} p<0.01$

release leptin. It has reported the level of leptin may represent the lipid content present in the body and increases the glucose metabolism [9-11]. Our results showed that leptin was significantly higher in mice fed with HFD than in the normal diet mice. BBD administration could restore the expression of leptin to normal level. These indicated BBD significantly reduced leptin levels in HFDinduced obese mice.

Hepatic de novo lipogenesis plays an important role in the development of NAFLD. The main pathway that regulates the initiation of fatty acid metabolism in the liver involves stimulation of SREBP-1c expression, a critical factor involved in hepatic lipid synthesis. The SREBP-1c promoter contains LXR response elements, which plays a key role in the activation of SREBP-1c transcription [24]. Mature SREBP-1c (the active form) translocates to the nucleus and promotes fatty acid biosynthesis via upregulating the expression of lipogenesis-related genes, including ACC and SCD-1 [25]. ACC active will produce malonyl-CoA blocking the build of new fatty acids and inhibiting the transfer of the fatty acyl group from acyl CoA to carnitine with carnitine acyltransferase [26]. SCD-1 is responsible for fatty acid desaturation $[27,28]$. CD36 also known as fatty acid translocase, contributes to intracellular lipid accumulation [29, 30]. We confirmed the genes of SREBP1-c, ACC, SCD-1, LXR $\alpha$ and CD36 
were up-regulation expression in HFD-fed mice than in the normal diet mice. And BBD treatment decreased the mRNA expression of these genes related to lipogenesis. PPAR- $\alpha$ is considered the primary factor regulating the $\beta$-oxidation of fatty acids, which limits the storage of liver fat [31]. Carnitine palmitoyl transferase enzyme 1 (CPT1 ) is one of the activators of PPAR- $\alpha$ expressed, which promotes the uptake of fat and mitochondrial fatty acid oxidation [32]. Our data showed BBD up-regulated the expression of $\beta$-oxidation-related genes of BBD + HFD group compared with untreated HFD group. These data indicated that BBD could attenuate de novo lipogenesis and accelerate the lipid metabolism, reducing hepatic lipid accumulation.

Previous study had showed that AMPK (adenosine monophosphate-activated protein kinase) signaling regulates lipid formation and promotes glucose uptake and fatty acid oxidation in adipose tissues [33]. On the one hand, the activation of AMPK phosphorylation decreases lipid synthesis by modulating the activities of various enzymes [11, 34]. On the other, hepatic AMPK would switch off SREBP-1c and increase inactivation of ACC and others $[12,35,36]$. Our results showed the expression of AMPK and p-AMPK were decreased in mouse of HFD group, suggested that the expression of p-AMPK in liver could be reduced by long term high fat diet. BBD increased the expression of AMPK and p-AMPK compared with untreated HFD group, which suggested BBD alleviated hepatic lipogenesis by activating AMPK pathway.

\section{Conclusions}

In summary, to the best of our knowledge this study is the first to find BBD supplementation protects against high fat diet-induced fat accumulation and non-alcoholic fatty liver disease by activation of AMPK pathway.

\begin{abstract}
Abbreviations
BBD: Babaodan; NAFLD: non-alcoholic fatty liver disease; HFD: the high-fat diet; TC: total cholesterol; TG: triglyceride; HDL-C: high density lipoprotein cholesterol; LDL-C: low density lipoprotein cholesterol; ALT: alanine aminotransferase; AST: aspartate aminotransferase; LFD: low-fat diet; SREBP1-c: sterol regulatory element-binding protein 1C; ACC: acetyl coenzyme A-carboxylase; SCD-1: stearoyl-CoA desaturase-1; LXRa: liver X receptor alpha; AMPK: adenosine monophosphate-activated protein kinase; CPT-1: carnitine palmitoyl transferase enzyme 1; PPAR-a: peroxisome proliferator-activated receptor alpha.
\end{abstract}

\section{Acknowledgements}

Not applicable.

\section{Authors' contributions}

LW, LZ and ZH designed the research; DS, SZ, GL, HZ, WL, JH, YJ, FY, QZ and RL performed the study; LZ, LW and ZH analyzed the data; DS, SZ, GL, LZ, LW and $\mathrm{ZH}$ wrote and revised the manuscript. All authors read and approved the final manuscript.

\section{Funding}

This project was supported by National Natural Science Foundation of China (Grant Nos. 81673641, 81630070, 81772940, 81872243); Special Funds for National Key Sci-Tech Special Project of China (Grant No. 2018ZX10723204005-004); and Shanghai Science and Technology Committee (Grant Nos. 14ZD1900403, 16JC 1405200); Science Fund for Creative Research Groups, NSFC, China (Grant No. 81521091).

\section{Availability of data and materials}

The datasets used and/or analyzed during the current study available from the corresponding author on reasonable request.

\section{Ethics approval and consent to participate}

All mouse experiments and procedures were approved by the Animal Ethics Committee of the Second Military Medical University, Shanghai, China, and all experiments were performed in accordance with the approved guidelines and regulations.

\section{Consent for publication}

Not applicable.

\section{Competing interests}

The authors declare that they have no competing interests.

\section{Author details}

${ }^{1}$ Tumor Immunology and Gene Therapy Center, Third Affiliated Hospital of Second Military Medical University, NO. 225 Changhai Road, Shanghai 200438, China. ${ }^{2}$ Department of Pharmacy, Changhai Hospital, Second Military Medical University, Shanghai 200433, China.

Received: 2 February 2019 Accepted: 9 September 2019

Published online: 18 September 2019

\section{References}

1. Gonzalez-Muniesa P, Martinez-Gonzalez MA, Hu FB, Despres JP, Matsuzawa Y, Loos RJF, et al. Obesity. Nat Rev Dis Primers. 2017;3:17034.

2. Ortega FB, Lavie CJ, Sui X. Health effects of overweight and obesity in 195 countries. N Engl J Med. 2017;377:1495.

3. Tanaka N, Kimura T, Fujimori N, Nagaya T, Komatsu M, Tanaka E. Current status, problems, and perspectives of non-alcoholic fatty liver disease research. World J Gastroenterol. 2019;25:163-77.

4. Younossi ZM, Koenig AB, Abdelatif D, Fazel Y, Henry L, Wymer M. Global epidemiology of nonalcoholic fatty liver disease-Meta-analytic assessment of prevalence, incidence, and outcomes. Hepatology. 2016;64:73-84

5. Ng M, Fleming T, Robinson M, Thomson B, Graetz N, Margono C, et al. Global, regional, and national prevalence of overweight and obesity in children and adults during 1980-2013: a systematic analysis for the Global Burden of Disease Study 2013. Lancet. 2014;384:766-81.

6. Zhu JZ, Zhou QY, Wang YM, Dai YN, Zhu J, Yu CH, et al. Prevalence of fatty liver disease and the economy in China: a systematic review. World J Gastroenterol. 2015;21:5695-706.

7. Song LB, Gao S, Zhang AQ, Qian X, Liu LM. Babaodan Capsule () combined with Qingyi Huaji Formula () in advanced pancreatic cancer-a feasibility study. Chin J Integr Med. 2017;23:937-42.

8. Liang L, Yang X, Yu Y, Li X, Wu Y, Shi R, et al. Babao Dan attenuates hepatic fibrosis by inhibiting hepatic stellate cells activation and proliferation via TLR4 signaling pathway. Oncotarget. 2016;7:82554-66.

9. Polyzos SA, Aronis KN, Kountouras J, Raptis DD, Vasiloglou MF, Mantzoros CS. Circulating leptin in non-alcoholic fatty liver disease: a systematic review and meta-analysis. Diabetologia. 2016;59:30-43.

10. O'Neill HM, Lally JS, Galic S, Thomas M, Azizi PD, Fullerton MD, et al. AMPK phosphorylation of ACC2 is required for skeletal muscle fatty acid oxidation and insulin sensitivity in mice. Diabetologia. 2014;57:1693-702.

11. Nepali S, Cha JY, Ki HH, Lee HY, Kim YH, Kim DK, et al. Chrysanthemum indicum Inhibits Adipogenesis and Activates the AMPK Pathway in HighFat-Diet-Induced Obese Mice. Am J Chin Med. 2018;46:119-36. 
12. Minokoshi Y, Kim YB, Peroni OD, Fryer LG, Muller C, Carling D, et al. Leptin stimulates fatty-acid oxidation by activating AMP-activated protein kinase. Nature. 2002;415:339-43.

13. Donnelly KL, Smith Cl, Schwarzenberg SJ, Jessurun J, Boldt MD, Parks EJ. Sources of fatty acids stored in liver and secreted via lipoproteins in patients with nonalcoholic fatty liver disease. J Clin Invest. 2005;115:1343-51.

14. Lumeng CN, Deyoung SM, Bodzin JL, Saltiel AR. Increased inflammatory properties of adipose tissue macrophages recruited during diet-induced obesity. Diabetes. 2007;56:16-23.

15. Clement K, Viguerie N, Poitou C, Carette C, Pelloux V, Curat CA, et al. Weight loss regulates inflammation-related genes in white adipose tissue of obese subjects. FASEB J. 2004;18:1657-69.

16. Hotamisligil GS. Inflammatory pathways and insulin action. Int J Obes Relat Metab Disord. 2003;27(Suppl 3):S53-5.

17. Odegaard II, Chawla A. Leukocyte set points in metabolic disease. F1000 Biol Rep. 2012; 4:13.

18. He W, Maedler K. Obesity is associated with a more inflammatory phenotype of macrophages in human pancreatic islets. Diabetologia. 2018:61:S256.

19. Zhang L, Wang CC. Inflammatory response of macrophages in infection. Hepatobiliary Pancreat Dis Int. 2014;13:138-52.

20. Wang TT, He CQ. Pro-inflammatory cytokines: the link between obesity and osteoarthritis. Cytokine Growth Factor Rev. 2018;44:38-50.

21. Xu B, Xie X. Neurotrophic factor control of satiety and body weight. Nat Rev Neurosci. 2016;17:282-92.

22. Pigeyre M, Yazdi FT, Kaur Y, Meyre D. Recent progress in genetics, epigenetics and metagenomics unveils the pathophysiology of human obesity. Clin Sci (Lond). 2016;130:943-86.

23. Nakazawa M, Obata Y, Nishino T, Abe S, Nakazawa Y, Abe K, et al. Involvement of leptin in the progression of experimentally induced peritoneal fibrosis in mice. Acta Histochem Cytochem. 2013;46:75-84.

24. Deng X, Zhang W, I OS, Williams JB, Dong Q, Park EA, et al. FoxO1 inhibits sterol regulatory element-binding protein-1c (SREBP-1c) gene expression via transcription factors Sp1 and SREBP-1c. J Biol Chem. 2012;287:20132-43.

25. Wang $M, M a ~ L J$, Yang $Y, X i a o ~ Z$, Wan JB. n-3 Polyunsaturated fatty acids for the management of alcoholic liver disease: a critical review. Crit Rev Food Sci Nutr. 2018;1:1-14.

26. Zachariah Tom R, Garcia-Roves PM, Sjogren RJ, Jiang LQ, Holmstrom MH, Deshmukh AS, et al. Effects of AMPK activation on insulin sensitivity and metabolism in leptin-deficient ob/ob mice. Diabetes. 2014;63:1560-71.
27. Stoeckman AK, Towle HC. The role of SREBP-1c in nutritional regulation of lipogenic enzyme gene expression. J Biol Chem. 2002;277:27029-35.

28. Han H, Qiu F, Zhao H, Tang H, Li X, Shi D. Dietary flaxseed oil prevents western-type diet-induced nonalcoholic fatty liver disease in apolipoprotein-e knockout mice. Oxid Med Cell Longev. 2017;2017:3256241.

29. Wang M, Zhang X, Ma LJ, Feng RB, Yan C, Su H, et al. Omega-3 polyunsaturated fatty acids ameliorate ethanol-induced adipose hyperlipolysis: a mechanism for hepatoprotective effect against alcoholic liver disease. Biochim Biophys Acta Mol Basis Dis. 2017:1863:3190-201.

30. Bujold K, Rhainds D, Jossart C, Febbraio M, Marleau S, Ong H. CD36mediated cholesterol efflux is associated with PPARgamma activation via a MAPK-dependent COX-2 pathway in macrophages. Cardiovasc Res. 2009;83:457-64.

31. Alves CC, Waitzberg DL, de Andrade LS, Dos Santos Aguiar L, Reis MB, Guanabara CC, et al. Prebiotic and synbiotic modifications of beta oxidation and lipogenic gene expression after experimental hypercholesterolemia in rat liver. Front Microbiol. 2017;8:2010

32. Kersten S, Seydoux J, Peters JM, Gonzalez FJ, Desvergne B, Wahli W. Peroxisome proliferator-activated receptor alpha mediates the adaptive response to fasting. J Clin Invest. 1999;103:1489-98.

33. Richter EA, Hargreaves M. Exercise, GLUT4, and skeletal muscle glucose uptake. Physiol Rev. 2013;93:993-1017.

34. Daval M, Foufelle F, Ferre P. Functions of AMP-activated protein kinase in adipose tissue. J Physiol. 2006;574:55-62.

35. Li Y, Xu S, Mihaylova MM, Zheng B, Hou X, Jiang B, et al. AMPK phosphorylates and inhibits SREBP activity to attenuate hepatic steatosis and atherosclerosis in diet-induced insulin-resistant mice. Cell Metab. 2011;13:376-88.

36. Shen Z, Liang X, Rogers CQ, Rideout D, You M. Involvement of adiponectin-SIRT1-AMPK signaling in the protective action of rosiglitazone against alcoholic fatty liver in mice. Am J Physiol Gastrointest Liver Physiol. 2010;298:G364-74

\section{Publisher's Note}

Springer Nature remains neutral with regard to jurisdictional claims in published maps and institutional affiliations.
Ready to submit your research? Choose BMC and benefit from:

- fast, convenient online submission

- thorough peer review by experienced researchers in your field

- rapid publication on acceptance

- support for research data, including large and complex data types

- gold Open Access which fosters wider collaboration and increased citations

- maximum visibility for your research: over 100M website views per year

At $\mathrm{BMC}$, research is always in progress.

Learn more biomedcentral.com/submissions 